\title{
A Dataset for Benchmarking Time-Resolved Non-Line-of-Sight Imaging
}

\author{
Miguel Galindo \\ mjgalindo@unizar.es \\ Universidad de Zaragoza, I3A \\ Gordon Wetzstein \\ gordon.wetzstein@stanford.edu \\ Stanford University
}

\author{
Julio Marco \\ juliom@unizar.es \\ Universidad de Zaragoza, I3A \\ Diego Gutierrez \\ diegog@unizar.es \\ Universidad de Zaragoza, I3A
}

\author{
Matthew O'Toole \\ mpotoole@cmu.edu \\ Carnegie Mellon University \\ Adrian Jarabo \\ ajarabo@unizar.es \\ Universidad de Zaragoza, I3A
}

\begin{abstract}
Time-resolved imaging has made it possible to look around corners by exploiting information from diffuse light bounces. While there have been successive improvements in the field since its conception, so far it has only been proven to work in very simple and controlled scenarios. We present a public dataset of synthetic timeresolved Non-Line-of-Sight (NLOS) scenes with varied complexity aimed at benchmarking reconstructions. It includes scenes that are common in the real world but remain a challenge for NLOS reconstruction methods due to the ambiguous nature of higher-order diffuse bounces naturally occurring in them. With over 300 reconstructible scenes, the dataset contains an order of magnitude more scenes than what is available currently. The final objective of the dataset it to boost NLOS research to take it closer to its real-world applications.
\end{abstract}

\section{CCS CONCEPTS}

- Computing methodologies $\rightarrow$ Reconstruction; Computational photography; Ray tracing.

\section{KEYWORDS}

datasets, non-line-of-sight imaging, transient rendering

\section{ACM Reference Format:}

Miguel Galindo, Julio Marco, Matthew O’Toole, Gordon Wetzstein, Diego Gutierrez, and Adrian Jarabo. 2019. A Dataset for Benchmarking TimeResolved Non-Line-of-Sight Imaging. In Proceedings of SIGGRAPH '19 Posters. ACM, New York, NY, USA, 2 pages. https://doi.org/10.1145/3306214.3338583

\section{INTRODUCTION}

Time-resolved imaging partially disambiguates diffuse light paths, making it possible to recover information from surfaces beyond the line of sight of the imaging device [Jarabo et al. 2017]. This effectively allows us to look around corners [Kirmani et al. 2009], a technology with multiple applications such as security, spatial exploration, autonomous driving, or rescue operations.

Permission to make digital or hard copies of part or all of this work for personal or classroom use is granted without fee provided that copies are not made or distributed for profit or commercial advantage and that copies bear this notice and the full citation on the first page. Copyrights for third-party components of this work must be honored For all other uses, contact the owner/author(s).

SIGGRAPH '19 Posters, fuly 28 - August 01, 2019, Los Angeles, CA, USA

(c) 2019 Copyright held by the owner/author(s).

ACM ISBN 978-1-4503-6314-3/19/07.

https://doi.org/10.1145/3306214.3338583
Typically, Non-Line-of-Sight imaging works with light paths formed by three bounces, first on a visible diffuse wall, second on a hidden surface and third back on the initial wall (see Figure 1 left). Many works have successfully exploited this information to obtain 3D representations of hidden parts of scenes [Arellano et al. 2017; Buttafava et al. 2015; Heide et al. 2018, 2014; O'Toole et al. 2018; Velten et al. 2012]. However, the scenarios used to validate them so far have been very simple; most often isolated planar diffuse objects in controlled environments. This kind of setup has been used extensively to highlight third-bounce information and obtain cleaner reconstructions. However, the real world is rarely that simple. Indirect light from multiple visible and hidden surfaces contribute to the time-resolved image, causing artifacts in their reconstructions.

Other computer vision tasks have proven that having readily accessible, labeled data and error metrics can be essential to improve the effectiveness of reconstruction methods, including other problems based on time-resolved data [Butler et al. 2012; Guo et al. 2018; Marco et al. 2017]. Klein and colleagues [2018] released a synthetic dataset and benchmark platform for various NLOS reconstruction problems, including geometry reconstruction. For this problem, the dataset contains 16 scenes entirely made from hidden objects floating in isolation, following the canonical setups used in previous works. These are too few and simple to obtain deeper insights from them or attempt data-driven approaches in NLOS imaging. In this work, we present a new synthetic dataset for NLOS reconstruction (see Figure 1), that contains an order of magnitude more examples and includes complex scenarios with challenging light transport conditions.

\section{OUR DATASET}

Inspired by these efforts, we release a synthetic dataset of timeresolved images from 86 distinct NLOS scenes. Considering variations in BRDFs and capture parameters, the dataset will contain around 300 time-resolved images for hidden geometry reconstruction, surpassing the quantities in previous datasets. In contrast with previous works, our goal is to cover a wider range of scenarios, from simple environments containing complex geometry, to complex real-world use-cases (see Figure 2), opening the field to data-driven works while providing an accessible means for benchmarking existing ones and simplifying the work of researchers. We consider two types of scenes separately: those with single hidden objects in canonical NLOS setups, and real-world-like scenes of indoor and outdoor spaces. The simpler scenes being useful to quickly 


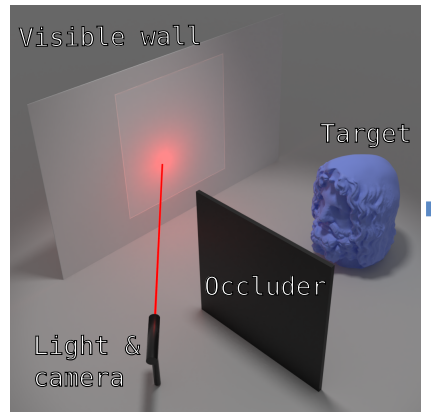

Input NLOS scene

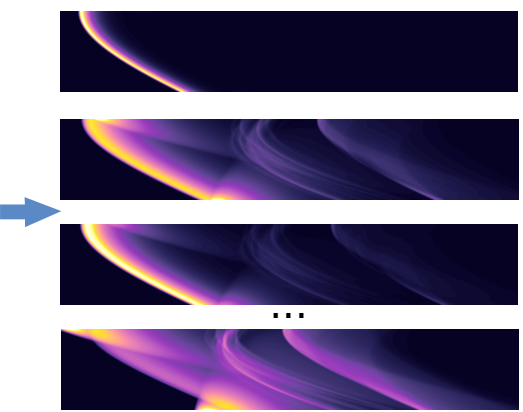

Simulated time-resolved responses

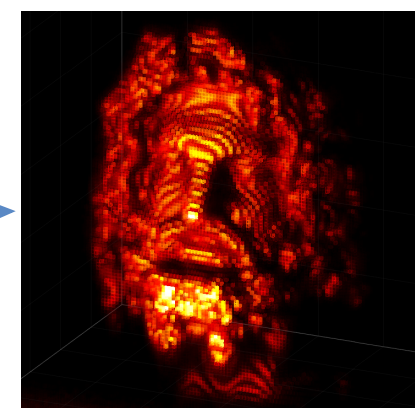

Reconstruction

Figure 1: The input scenes contain a virtual capture and illumination device aiming at a visible wall or planar surface. We then simulate a grid of illumination and capture points on this surface to produce time-resolved images. Reconstruction algorithms can then use this time-resolved data to obtain voxelized reconstructions of the hidden scene.

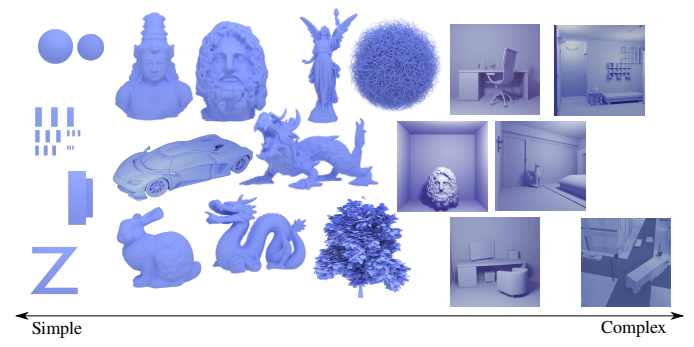

Figure 2: Geometry and scenes from our dataset ordered on a range from simple shapes to real-world scenarios. Our simplest scenes are similar to those in previous works, using isolated planes or simple shapes. We progressively increase the complexity of the scenes by including shapes with high frequency details, concave and convex surfaces, and placing them in environments that contribute multibounce interreflections further increasing reconstruction difficulty.

diagnose reconstruction problems and benchmark methods to compare with other works. The more realistic scenes are much more challenging and offer better approximations of the performance of a reconstruction method when applied in the real world.

To increase the generality and variety of the dataset, each simulation uses a different high-resolution pattern for the illumination and capture of a visible planar wall, all containing 65,536 illumination and capture pairs. The simulations are made leveraging the recent advances on transient light transport simulation by Jarabo and colleagues [2014]. To facilitate research, each light bounce is stored independently in the dataset, making it possible to work on the simpler three-bounce case first, and then on minimizing the effects of the additional light bounces on the reconstructions.

All data is already available on-line ${ }^{1}$. We hope this dataset will become an integral part in the development and testing process of new algorithms for NLOS imaging.

\section{ACKNOWLEDGMENTS}

This project has been funded by DARPA (project REVEAL), the European Research Council (ERC) under the EU's Horizon 2020 research and innovation programme (project CHAMELEON, grant No 682080), the Spanish Ministry of Economy and Competitiveness (project TIN2016-78753-P), and the BBVA Foundation through a Leonardo grant.

\section{REFERENCES}

Victor Arellano, Diego Gutierrez, and Adrian Jarabo. 2017. Fast Back-Projection for Non-Line of Sight Reconstruction. Optics Express 25, 10 (2017).

D. J. Butler, J. Wulff, G. B. Stanley, and M. J. Black. 2012. A naturalistic open source movie for optical flow evaluation. In The European Conference on Computer Vision $(E C C V)$, A. Fitzgibbon et al. (Eds.) (Ed.). Springer-Verlag.

Mauro Buttafava, Jessica Zeman, Alberto Tosi, Kevin Eliceiri, and Andreas Velten. 2015. Non-line-of-sight imaging using a time-gated single photon avalanche diode. Optics Express 23, 16 (Aug 2015), 20997-21011.

Qi Guo, Iuri Frosio, Orazio Gallo, Todd Zickler, and Jan Kautz. 2018. Tackling 3D ToF Artifacts Through Learning and the FLAT Dataset. In The European Conference on Computer Vision (ECCV).

Felix Heide, Matthew O'Toole, Kai Zang, David Lindell, Steven Diamond, and Gordon Wetzstein. 2018. Non-line-of-sight Imaging with Partial Occluders and Surface Normals. arXiv:cs.CV/1711.07134 arXiv:1711.07134.

Felix Heide, Lei Xiao, Wolfgang Heidrich, and Matthias B. Hullin. 2014. Diffuse Mirrors: 3D Reconstruction from Diffuse Indirect Illumination Using inexpensive Time-ofFlight Sensors. In IEEE International Conference on Computer Vision and Pattern Recognition (CVPR)

Adrian Jarabo, Julio Marco, Adolfo Muñoz, Raul Buisan, Wojciech Jarosz, and Diego Gutierrez. 2014. A Framework for Transient Rendering. ACM Transactions on Graphics (SIGGRAPH Asia 2014) 33, 6 (2014).

Adrian Jarabo, Belen Masia, Julio Marco, and Diego Gutierrez. 2017. Recent Advances in Transient Imaging: A Computer Graphics and Vision Perspective. Visual Informatics 1,1 (2017).

Ahmed Kirmani, Tyler Hutchison, James Davis, and Ramesh Raskar. 2009. Looking around the corner using transient imaging. In Computer Vision, 2009 IEEE 12th International Conference on. IEEE, 159-166.

Jonathan Klein, Martin Laurenzis, Dominik L. Michels, and Matthias B. Hullin. 2018. A Quantitative Platform for Non-Line-of-Sight Imaging Problems. In British Machine Vision Conference 2018 (BMVC). 104.

Julio Marco, Quercus Hernandez, Adolfo Munoz, Yue Dong, Adrian Jarabo, Min Kim, Xin Tong, and Diego Gutierrez. 2017. DeepToF: Off-the-Shelf Real-Time Correction of Multipath Interference in Time-of-Flight Imaging. ACM Transactions on Graphics (SIGGRAPH Asia 2017) 36, 6 (2017).

Matthew O'Toole, David B. Lindell, and Gordon Wetzstein. 2018. Confocal Non-Lineof-Sight Imaging Based on the Light-Cone Transform. Nature (2018).

Andreas Velten, Thomas Willwacher, Otkrist Gupta, Ashok Veeraraghavan, Moungi G Bawendi, and Ramesh Raskar. 2012. Recovering three-dimensional shape around a corner using ultrafast time-of-flight imaging. Nature communications 3 (2012), 745. 